\title{
Influence of Varying Temperature and Concentration on MHD Peristaltic Transport for Jeffrey Fluid with Variable Viscosity through Porous Channel
}

\author{
Ahmed A .Hussein Al-Aridhee a Dheia G. Salih Al-Khafajy ${ }^{b}$ \\ a Department of Mathematics, faculty of Computer Science and Information Technology, University of Al-Qadisiyah, Diwaneyah, Iraq. \\ Email:ahmedalardy845@gmail.com \\ ${ }^{b}$ Department of Mathematics, faculty of Computer Science and Information Technology, University of Al-Qadisiyah, Diwaneyah, Iraq. E. mail: \\ dr.dheia.g.salih@gmail.com
}

\section{A RT ICLE INFO}

\section{Article history:}

Received: 25 /04/2019

Rrevised form: $26 / 05 / 2019$

Accepted : $02 / 06 / 2019$

Available online: $17 / 06 / 2019$

\section{Keywords:}

Magneto hydrodynamic, Jeffrey Fluid, Variable Viscosity, heat transfer, Porous Channel.

\begin{abstract}
A B S T RAC T
The present paper deals with the peristaltic motion of Jeffrey fluid with varying temperature and concentration through a porous medium in a coaxial uniform circular tube. The fluid is assumed to be non-Newtonian, namely Jeffrey fluid. The inner tube is uniform, while the outer flexible tube has a sinusoidal wave traveling down its wall. The analytical formulas of the velocity and temperature have been obtained in terms of the Bessel function of first and second kinds. The numerical formula of the axial velocity, temperature and concentration are obtained as functions of the physical parameters of the problem (Darcy number, magnetic parameter, thermal Grashof number, Reynolds number, Prandtl number, and Schmidt number) with other physical parameters are obtained. The Influence of physical parameters of the problem on this formula are discussed numerically and illustrated graphically through a set of figures.
\end{abstract}

\section{Introduction}

Peristalsis is a mechanism to pump the fluid by means of moving contraction on the channel walls. This process has quite useful applications in many biological systems and industry, it occurs in swallowing food through the esophagus, chyme motion in the gastrointestinal tract, the vaso motion of small blood vessels such as venules, capillaries , and arterioles, urine transport from kidney to bladder, sanitary fluid transport of corrosive fluids, a toxic liquid transport in the nuclear industry, and so forth. In view of such physiological and industrial applications, the peristaltic flows has been studied with great interest by the various researchers for viscous and non-Newtonian fluids [6], [11], [2]. Viscosity is one of the most important specifications in fluids. It is a very effective factor in the transfer and movement of blood within veins, arteries, blood vessels and capillaries in the human body and animals. It is also important in the process of oil production, which determines the flow of reservoir fluids through the pores found in rocks containing oil. Viscosity is of great importance in the fields of industrial chemistry, food, beverages, paints, printing, organic chemistry, the environment and so on. There are many studies in the scientific literature on

Corresponding author Ahmed A.Hussein Al-Aridhee 
fluid movement in the channel, for examples; the effect of heat transfer on the (MHD) oscillatory flow of a Jeffrey fluid with variable viscosity model through porous medium studied by Al-Khafajy [1]. M. Vidhya [12] studied the flow of blood through the veins and arteries. [8] Variable viscosity was studied through porosity the medium method of homotopy analysis is used to solve problem, [5] effect of Heat Transfer on the Oscillation Flow of Hydrodynamical Magnetism of Williamson Fluid through Porosity average, [4] , [3] They studied the variable viscosity during a porous medium ,flows through a porous medium occur in filtration of fluids. Several investigations have been published by using generalized Darcy's law where the convective acceleration and viscous stress are taken into account [9], [10], [5]. The study considers a mathematical model for the influence of varying temperature and concentration on MHD peristaltic Transport for Jeffrey fluid with variable viscosity through Porous channel. The study uses the perturbation technique series to solve the problem. The results of the physical parameter problem are discussed by using the graphs.

\section{Mathematical Formulation}

Consider a peristaltic flow of an incompressible Jeffrey fluid in a coaxial uniform circular tube. The cylindrical coordinates are considered, where $R$ is along the radius of the tube and $Z$ coincides with the axes of the tube as shown in figure (1).

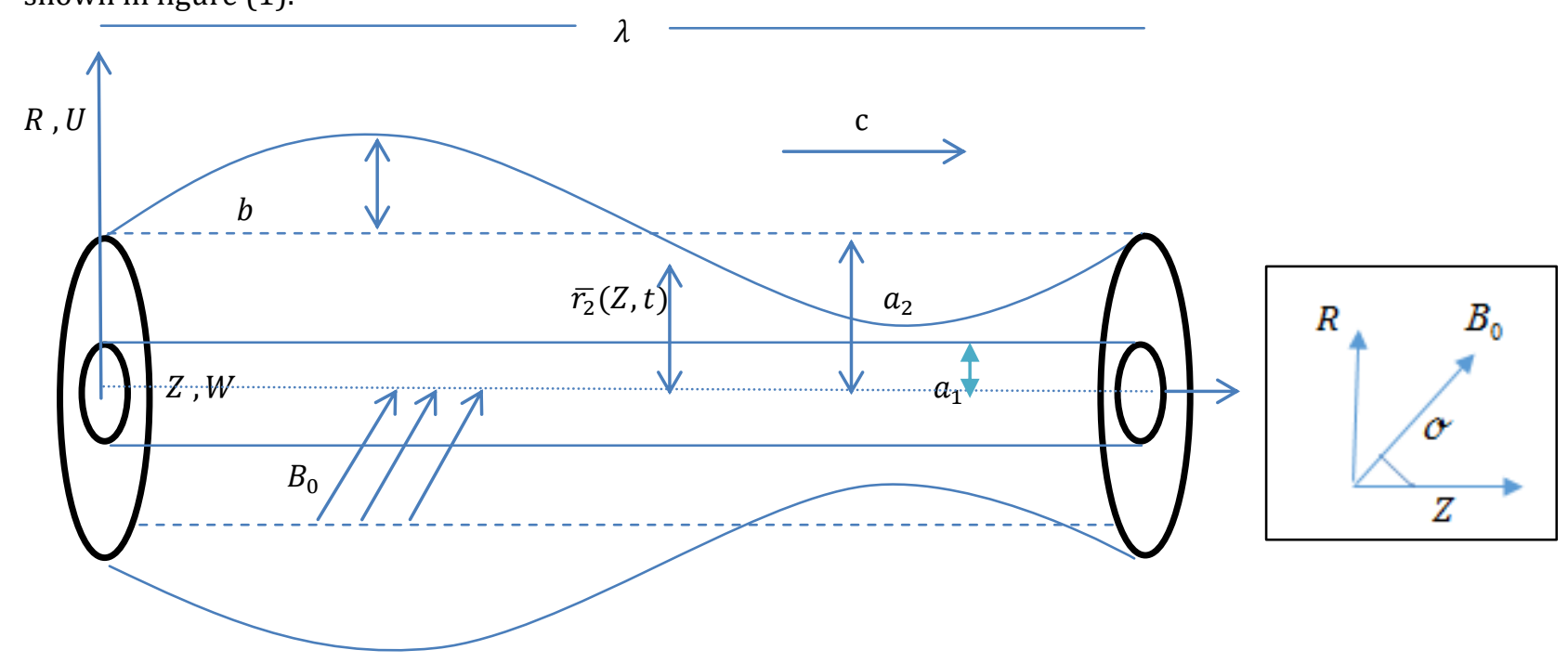

Fig. (1): Geometry of the problem.

The geometry of wall surface is described as:

$H(\bar{Z}, \bar{t})=a+b \sin \left[\frac{2 \pi}{\lambda}(\bar{Z}-c \bar{t})\right]$

where $a$ is the average radius of the undisturbed tube, $b$ is the amplitude of the peristaltic wave, $\lambda$ is the wavelength, $c$ is the wave propagation speed, and $\bar{t}$ is the time.

\section{Basic Equations}

The basic equations governing the non-Newtonian Jeffrey fluid are given by:

$\nabla \bar{V}=0$,

The momentum equation is given by:

$\rho(\bar{V} . \nabla) \bar{V}=\nabla \bar{\tau}+\mu_{e} \bar{J} \times \bar{B}-\frac{\mu(T)}{K^{*}} \bar{V}+\rho g \beta_{T}\left(T-T_{0}\right)+\rho g \beta_{C}\left(C-C_{0}\right)$,

The temperature equation is given by:

$c_{p} \cdot \rho(\bar{V} \cdot \nabla) T=K \cdot \nabla^{2} T-\nabla \cdot q_{r}-Q T$, 
The concentration equation is given by:

$(\bar{V} . \nabla) C=D_{m} \nabla^{2} C+\frac{D_{m} k_{T}}{T_{m}} \nabla^{2} T$.

Where $\nabla^{2}=\frac{1}{r} \frac{\partial}{\partial r}\left(r \frac{\partial}{\partial r}\right)$ (Laplace operator). Also $\bar{V}$ is the velocity, $\mu(T)$ is the variable viscosity, $K^{*}$ is the permeability, $\bar{B}=\left(0, B_{0}, 0\right)$ is the magnetic field, $\sigma$ is the electrical conductivity, $\mu_{e}$ is the magnetic permeability, and $\bar{\tau}$ is the Cauchy stress tensor. Also $T$ and $C$ are the temperature and concentration of the fluid, $K$ is the thermal conductivity, $c_{p}$ is the specific heat capacity at constant pressure, $D_{m}$ is the coefficient of mass diffusivity, $T_{m}$ is the mean fluid temperature and $k_{T}$ is the thermal diffusion ratio.

\section{Constitutive Equations}

The constitutive equations for an incompressible Jeffrey fluid are given by:

$$
\begin{aligned}
& \bar{\tau}=-\bar{P} \bar{I}+\bar{S}, \\
& \bar{S}=\frac{\mu(T)}{1+\lambda_{1}}\left(\bar{\gamma}+\lambda_{2} \bar{\gamma}\right),
\end{aligned}
$$

where $\bar{S}$ is the extra stress tensor, $\bar{P}$ is the pressure, $\bar{I}$ is the identity tensor, $\lambda_{1}$ is the ratio of relaxation to retardation times, $\overline{\dot{\gamma}}$ is the shear rate, and $\lambda_{2}$ is the retardation time.

\section{Method of solution}

Let $\bar{U}$ and $\bar{W}$ be the respective velocity components in the radial and axial directions in the fixed frame, respectively. For the unsteady two - dimensional flow, the velocity components may be written follows:

$V=(\bar{U}(\bar{r}, \bar{z}), 0, \bar{W}(\bar{r}, \bar{z}))$.

The temperature and concentration functions may be written as follows:

$T=T(r, z)$, and $C=C(r, z)$.

The equations of motion (2) - (7) and the constitutive relations (8), (9) take the form

$\frac{\partial \bar{U}}{\partial \bar{R}}+\frac{\bar{U}}{\bar{R}}+\frac{\partial \bar{W}}{\partial \bar{Z}}=0$,

$\rho\left(\frac{\partial \bar{U}}{\partial \bar{t}}+\bar{U} \frac{\partial \bar{U}}{\partial \bar{R}}+\bar{W} \frac{\partial \bar{U}}{\partial \bar{Z}}\right)=-\frac{\partial \bar{p}}{\partial \bar{R}}+\frac{1}{\bar{R}} \frac{\partial}{\partial \bar{R}}\left(\bar{R} \bar{S}_{\bar{R} \bar{R}}\right)+\frac{\partial}{\partial \bar{Z}}\left(\bar{S}_{\bar{R} \bar{Z}}\right)-\frac{\bar{S}_{\bar{\vartheta} \bar{\vartheta}}}{\bar{R}}-\frac{\mu(T)}{k} \bar{U}$,

$\rho\left(\frac{\partial \bar{W}}{\partial \bar{t}}+\bar{U} \frac{\partial \bar{W}}{\partial \bar{R}}+\bar{W} \frac{\partial \bar{W}}{\partial \bar{Z}}\right)=-\frac{\partial \bar{p}}{\partial \bar{Z}}+\frac{1}{\bar{R}} \frac{\partial}{\partial \bar{R}}\left(\bar{R} \bar{S}_{\bar{R} \bar{Z}}\right)+\frac{\partial}{\partial \bar{Z}}\left(\bar{S}_{\bar{Z} \bar{Z}}\right)+\rho g \beta_{T}\left(T-T_{0}\right)+\rho g \beta_{C}\left(C-C_{0}\right)-\sigma B_{0}^{2} \operatorname{Sin}^{2}(\sigma) \bar{W}-\frac{\mu(T)}{k} \bar{W}$

$\frac{\partial T}{\partial \bar{t}}+\bar{U} \frac{\partial T}{\partial \bar{R}}+\bar{W} \frac{\partial T}{\partial \bar{Z}}=\frac{K}{c_{p} \rho}\left(\frac{\partial^{2} T}{\partial \bar{R}^{2}}+\frac{1}{\bar{R}} \frac{\partial T}{\partial \bar{R}}+\frac{\partial^{2} T}{\partial \bar{Z}^{2}}\right)-\frac{16 \sigma_{0} T_{2}^{E}}{3 k_{0} c_{p} \rho} \frac{1}{\bar{R}} \frac{\partial}{\partial \bar{R}}\left(\bar{R} \frac{\partial T}{\partial \bar{R}}\right)-\frac{Q}{c_{p} \rho} T$,

$\frac{\partial C}{\partial \bar{t}}+\bar{U} \frac{\partial C}{\partial \bar{R}}+\bar{W} \frac{\partial C}{\partial \bar{Z}}=D_{m}\left(\frac{\partial^{2} C}{\partial \bar{R}^{2}}+\frac{1}{\bar{R}} \frac{\partial C}{\partial \bar{R}}+\frac{\partial^{2} C}{\partial \bar{Z}^{2}}\right)+\frac{D_{m} k_{T}}{T_{m}}\left(\frac{\partial^{2} T}{\partial \bar{R}^{2}}+\frac{1}{\bar{R}} \frac{\partial T}{\partial \bar{R}}+\frac{\partial^{2} T}{\partial \bar{Z}^{2}}\right)$.

In the fixed coordinates $(\bar{R}, \bar{Z})$ the flow between the two tubes is unsteady. It becomes steady in a wave frame $(r, z)$ moving with the same speed as wave in the $Z$-direction. The transformation between the two frames is:

$\bar{r}=\bar{R}, \bar{z}=\bar{Z}-c \bar{t}$,

$\bar{u}=\bar{U}, \bar{w}=\bar{W}+\mathrm{c}$,

Where $(\bar{r}, \bar{z})$ and $(\bar{U}, \bar{W})$ are the velocity components in the moving and fixed frames, respectively. After using these transformations, the equations of motion are;

$\left(\frac{\partial \bar{u}}{\partial \bar{r}}+\frac{\bar{u}}{\bar{r}}+\frac{\partial \bar{w}}{\partial \bar{z}}\right)=0$,

$\rho\left(\bar{u} \frac{\partial \bar{u}}{\partial \bar{r}}+\bar{w} \frac{\partial \bar{u}}{\partial \bar{z}}\right)=-\frac{\partial \bar{p}}{\partial \bar{r}}+\frac{1}{\bar{r}} \frac{\partial}{\partial \bar{r}}\left(\bar{r} S_{\bar{r} \bar{r}}\right)+\frac{\partial}{\partial \bar{z}}\left(S_{\bar{r} \bar{z}}\right)-\frac{S_{\bar{\vartheta} \bar{\vartheta}}}{\bar{r}}-\frac{\mu(T)}{k} \bar{u}$,

$\rho\left(\bar{u} \frac{\partial \bar{w}}{\partial r}+\bar{w} \frac{\partial \bar{w}}{\partial z}\right)=-\frac{\partial \bar{p}}{\partial \bar{z}}+\frac{1}{\bar{r}} \frac{\partial}{\partial \bar{r}}\left(\bar{r} S_{\bar{r} \bar{z}}\right)+\frac{\partial}{\partial \bar{z}}\left(S_{\bar{z} \bar{z}}\right)+\rho g \beta_{T}\left(T-T_{0}\right)+\rho g \beta_{C}\left(C-C_{0}\right)-\sigma B_{0}^{2} \operatorname{Sin}^{2}(\sigma) \bar{w}-\frac{\mu(T)}{k} \bar{w}$,

$\frac{\partial T}{\partial \bar{t}}+\bar{u} \frac{\partial T}{\partial \bar{r}}+\bar{w} \frac{\partial T}{\partial \bar{z}}=\frac{K}{c_{p} \rho}\left(\frac{\partial^{2} T}{\partial \bar{r}^{2}}+\frac{1}{\bar{r}} \frac{\partial T}{\partial \bar{r}}+\frac{\partial^{2} T}{\partial \bar{z}^{2}}\right)-\frac{16 \sigma_{0} T_{2}^{E}}{3 k_{0} c_{p} \rho} \frac{1}{\bar{r}} \frac{\partial}{\partial \bar{r}}\left(\bar{r} \frac{\partial T}{\partial \bar{r}}\right)-\frac{Q}{c_{p} \rho} T$,

$\frac{\partial C}{\partial \bar{t}}+\bar{U} \frac{\partial C}{\partial \bar{R}}+\bar{W} \frac{\partial C}{\partial \bar{Z}}=D_{m}\left(\frac{\partial^{2} C}{\partial \bar{R}^{2}}+\frac{1}{\bar{R}} \frac{\partial C}{\partial \bar{R}}+\frac{\partial^{2} C}{\partial \bar{Z}^{2}}\right)+\frac{D_{m} k_{T}}{T_{m}}\left(\frac{\partial^{2} T}{\partial \bar{R}^{2}}+\frac{1}{\bar{R}} \frac{\partial T}{\partial \bar{R}}+\frac{\partial^{2} T}{\partial \bar{Z}^{2}}\right)$. 
Where $\bar{u}$ and $\bar{w}$ are the velocity components in the $\bar{r}$ and $\bar{z}$ directions, respectively, $\rho$ is the density, $\bar{p}$ is the pressure, $\mu$ is the viscosity. In order to simplify the governing equations of the motion, we may introduce the following dimensionless transformations as follows:

$$
\left.\begin{array}{c}
S=\frac{a_{2} \bar{S}}{\mu c}, \mu=\frac{\mu(T)}{\mu(\vartheta)}, r=\frac{\bar{r}}{a_{2}}, z=\frac{\bar{z}}{\lambda}, \delta=\frac{a_{2}}{\lambda}, u=\frac{\lambda \bar{u}}{a_{2} c}, w=\frac{\bar{w}}{c}, \vartheta=\frac{T-T_{0}}{T_{1}-T_{0}}, \varphi=\frac{C-C_{0}}{C_{1}-C_{0}}, R_{n}=\frac{\rho K_{0} C_{p} v}{4 T_{2}^{2} \sigma_{0}}, r_{1}=\frac{\overline{r_{1}}}{a_{2}}=\varepsilon \\
D a=\frac{K}{a_{2}^{2}}, P_{r}=\frac{\mu c_{p}}{K}, M_{\sigma}^{2}=\frac{\sigma B_{0}^{2}}{\mu} \sin ^{2}(\sigma) a_{2}^{2}, R_{e}=\frac{\rho c a_{2}}{\mu}, \emptyset=\frac{b}{a_{2}}, r_{2}=\frac{\overline{r_{2}}}{a_{2}}=1+\emptyset \sin (2 \pi \bar{z}), G_{c}=\frac{\rho g \beta_{c} a_{2}^{2}\left(C_{1}-C_{0}\right)}{\mu c} \\
S_{c}=\frac{\mu c p}{K}, S_{r}=\frac{D_{m} k_{T}\left(T_{1}-T_{0}\right)}{T_{m}\left(C_{1}-C_{0}\right)}, G_{r}=\frac{\rho g \beta_{T} a_{2}^{2}\left(T_{1}-T_{0}\right)}{\mu c}, p=\frac{a_{2}^{2} \bar{p}}{\mu \lambda c}
\end{array}\right\}
$$

where $\varnothing$ is the amplitude ratio, $R_{e}$ the Reynolds number, $D a$ the Darcy number, $S_{r}$ the Soret number, $R_{n}$ the Radiation parameter, $S_{c}$ the Schmidt number, $M_{\sigma}^{2}$ the magnetic parameter and $\delta$ is the dimensionless wave number. Substituting (22) into equations (17) - (21), we have:

$\left(\frac{\partial u}{\partial r}+\frac{u}{r}+\frac{\partial w}{\partial z}\right)=0$

$R_{e} \delta^{3}\left(u \frac{\partial u}{\partial r}+w \frac{\partial u}{\partial z}\right)=-\frac{\partial p}{\partial r}+\delta \frac{1}{r} \frac{\partial}{\partial r}\left(r S_{r r}\right)+\delta^{2} \frac{\partial}{\partial z}\left(S_{r z}\right)-\delta \frac{S_{\vartheta \vartheta}}{r}-\frac{a_{2}}{k} \delta^{2} u$,

$R_{e} \delta\left(u \frac{\partial w}{\partial r}+w \frac{\partial w}{\partial z}\right)=-\frac{\partial p}{\partial z}+\frac{1}{r} S_{r z}+\frac{\partial}{\partial r}\left(S_{r z}\right)+\delta \frac{\partial}{\partial z}\left(S_{z z}\right)-\left(M_{1}^{2}+\frac{\mu(\vartheta)}{D_{a}}\right) w+G r \vartheta+G c \varphi-\left(M_{\sigma}^{2}+\frac{\mu(\vartheta)}{D_{a}}\right)$

$\delta\left(u \frac{\partial \vartheta}{\partial r}+w \frac{\partial \vartheta}{\partial z}\right)=\frac{1}{p_{r}}\left(\frac{\partial^{2} \vartheta}{\partial r^{2}}+\frac{1}{r} \frac{\partial \vartheta}{\partial r}+\delta^{2} \frac{\partial^{2} \vartheta}{\partial z^{2}}\right)+\frac{4}{3 R_{n}} \frac{1}{r} \frac{\partial}{\partial r}\left(r \frac{\partial \vartheta}{\partial r}\right)-\Omega \vartheta$,

$\delta\left(u \frac{\partial \varphi}{\partial r}+w \frac{\partial \varphi}{\partial z}\right)=\frac{1}{S_{c}}\left(\frac{\partial^{2} \varphi}{\partial r^{2}}+\frac{1}{r} \frac{\partial \varphi}{\partial r}+\delta^{2} \frac{\partial^{2} \varphi}{\partial z^{2}}\right)+S_{r}\left(\frac{\partial^{2} \vartheta}{\partial r^{2}}+\frac{1}{r} \frac{\partial \vartheta}{\partial r}+\delta^{2} \frac{\partial^{2} \vartheta}{\partial z^{2}}\right)$.

where

$S_{r r}=\frac{2 \delta \mu(\vartheta)}{1+\lambda_{1}}\left[1+\frac{c \lambda_{2} \delta}{a_{2}}\left(u \frac{\partial}{\partial r}+w \frac{\partial}{\partial z}\right)\left(\frac{\partial u}{\partial r}\right)\right]$,

$S_{r z}=\frac{\mu(\vartheta)}{1+\lambda_{1}}\left[1+\frac{c \lambda_{2} \delta}{a_{2}}\left(u \frac{\partial}{\partial r}+w \frac{\partial}{\partial z}\right)\left(\frac{\partial w}{\partial r}+\delta^{2} \frac{\partial u}{\partial z}\right)\right]$,

$S_{\vartheta \vartheta}=\frac{2 \delta \mu(\vartheta)}{1+\lambda_{1}}\left[\frac{c \lambda_{2} \delta}{a_{2}}\left[\frac{u}{r} \frac{\partial u}{\partial r}-\frac{u^{2}}{r^{2}}+\frac{w}{r} \frac{\partial u}{\partial z}\right]\right.$,

$S_{z Z}=\frac{2 \delta \mu(\vartheta)}{1+\lambda_{1}}\left[1+\frac{c \lambda_{2} \delta}{a_{2}}\left(u \frac{\partial}{\partial r}+w \frac{\partial}{\partial z}\right)\left(\frac{\partial w}{\partial r}\right)\right]$,

the related boundary conditions regarding to the dimensionless variables in the wave frame are given by:

$w=-1, u=0, \vartheta=1, \varphi=1$ at $r=r_{1}=\varepsilon$

$w=-1, u=0, \vartheta=0, \varphi=0$ at $\left.r=r_{2}=1+\emptyset \cdot \operatorname{Sin}(2 \pi z)\right\}$

(32)

The general solution of the governing equations (22) - (26) in the general case seems to be impossible; therefore, we shall confine the analysis under the assumption of small dimensionless wave number. It follows that $\delta \ll 1$. In other words, we considered the long-wavelength approximation. Along to this assumption, equations (23) - (27) become:

$\left(\frac{\partial u}{\partial r}+\frac{u}{r}+\frac{\partial w}{\partial z}\right)=0$

$\frac{\partial p}{\partial r}=0$,

$\frac{\partial p}{\partial z}=\frac{1}{r} S_{r z}+\frac{\partial}{\partial r}\left(S_{r z}\right)-\left(M_{\sigma}^{2}+\frac{\mu(\vartheta)}{D_{a}}\right) w+G_{r} \vartheta+G_{c} \varphi-\left(M_{\sigma}^{2}+\frac{\mu(\vartheta)}{D_{a}}\right)$,

$\left(\frac{1}{R_{e} p_{r}}+\frac{4}{3 R_{n}}\right)\left(\frac{\partial^{2} \vartheta}{\partial r^{2}}+\frac{1}{r} \frac{\partial \vartheta}{\partial r}\right)-\Omega \vartheta=0$,

$\frac{1}{S_{c}}\left(\frac{\partial^{2} \varphi}{\partial r^{2}}+\frac{1}{r} \frac{\partial \varphi}{\partial r}\right)=-S_{r}\left(\frac{\partial^{2} \vartheta}{\partial r^{2}}+\frac{1}{r} \frac{\partial \vartheta}{\partial r}\right)$, 
Where $S_{r r}=S_{\vartheta \vartheta}=S_{z z}=0$ and $S_{r z}=\frac{\mu(\vartheta)}{1+\lambda 1}\left(\frac{\partial w}{\partial r}\right)$

Replacing $S_{r z}$ from equation (38) in equation (35), we have:

$\frac{\partial p}{\partial z}=\frac{1}{r} \frac{\mu(\vartheta)}{1+\lambda 1}\left(\frac{\partial w}{\partial r}\right)+\frac{\partial}{\partial r} \frac{\mu(\vartheta)}{1+\lambda 1}\left(\frac{\partial w}{\partial r}\right)-\left(M_{\sigma}^{2}+\frac{\mu(\vartheta)}{D_{a}}\right) w+G r \vartheta+G c \varphi-\left(M_{1}^{2}+\frac{\mu(\vartheta)}{D_{a}}\right)$,

\section{Solutions of the problem}

The temperature equation (36), can be written as;

$$
\left(\frac{\partial^{2} \vartheta}{\partial r^{2}}+\frac{1}{r} \frac{\partial \vartheta}{\partial r}\right)-\frac{\Omega}{\left(\frac{1}{R_{e} p_{r}}+\frac{4}{3 R_{n}}\right)} \vartheta=0
$$

The equation (40) takes the form:

$r^{2} \frac{\partial^{2} \vartheta}{\partial r^{2}}+r \frac{\partial \vartheta}{\partial r}-A r^{2} \vartheta=0$

after take $\frac{\Omega}{\left(\frac{1}{R_{e} p_{r}}+\frac{4}{3 R_{n}}\right)}=A$, which is the modified Bessel equation of order zero.

The general solution of equation (41) is

$$
\vartheta=d_{1} J_{0}[\sqrt{A} r]+d_{2} \mathrm{Y}_{0}[\sqrt{A} r]
$$

By using the boundary conditions Eq. (32), we have

$d_{1}=\frac{\mathrm{Y}_{0}[h \sqrt{A}]}{J_{0}[\epsilon \sqrt{A}] \mathrm{Y}_{0}[h \sqrt{A}]-J_{0}[h \sqrt{A}] \mathrm{Y}_{0}[\epsilon \sqrt{A}]}$

and $\quad d_{2}=\frac{J_{0}[h \sqrt{A}]}{J_{0}[h \sqrt{A}] \mathrm{Y}_{0}[\epsilon \sqrt{A}]-J_{0}[\epsilon \sqrt{A}] \mathrm{Y}_{0}[h \sqrt{A}]}$

The concentration equation (37), can be written as;

$\frac{1}{r} \frac{\partial}{\partial r}\left(r \frac{\partial \varphi}{\partial r}\right)=-S_{c} S_{r} \frac{1}{r} \frac{\partial}{\partial r}\left(r \frac{\partial \vartheta}{\partial r}\right)$

The general solution of equation (43) is

$\varphi=-\operatorname{ScSr} \vartheta+d_{3} \operatorname{Ln}[r]+d_{4}$

By using equation (42) and the boundary conditions given in equation (32), we have

$d_{3}=\frac{1+(S c S r)}{\operatorname{Ln}\left({ }^{1} / r_{2}\right)}$, and $d_{4}=-d_{3} \operatorname{Ln}\left(r_{2}\right)$.

Equation (34) shows that $p$ dependents on z only

\subsection{Reynold's Model of Viscosity:}

The Reynold's model and variation of viscosity with temperature are defined as:

$\mu(\vartheta)=e^{-\alpha \vartheta}$

By using the Maclaurin series, we get:

$\mu(\vartheta)=1-\alpha \vartheta, \alpha<<1$

$\frac{\partial p}{\partial z}=\frac{1}{r} \frac{1-\alpha \vartheta}{1+\lambda 1}\left(\frac{\partial w}{\partial r}\right)+\frac{\partial}{\partial r} \frac{1-\alpha \vartheta}{1+\lambda 1}\left(\frac{\partial w}{\partial r}\right)-\left(M_{\sigma}^{2}+\frac{1-\alpha \vartheta}{D_{a}}\right) w+G r \vartheta+G c \varphi-\left(M_{1}^{2}+\frac{1-\alpha \vartheta}{D_{a}}\right)$, 
Equation (44) is a non-linear differential equation and it is hard to find an exact solution, so will be used the perturbation technique to find the problem solution, as follows:

$w=w_{0}+\alpha w_{1}+\mathrm{O}\left(\alpha^{2}\right)$

$p=p_{0}+\alpha p_{1}+\mathrm{O}\left(\alpha^{2}\right)$

By substituting equations (48) and (49) into Eq. (47) with boundary conditions (32), we equalize the powers of $(\alpha)$, we get:

$\frac{\partial\left(p_{0}+\alpha p_{1}\right)}{\partial z}=\frac{1}{r} \frac{1-\alpha \vartheta}{1+\lambda 1}\left(\frac{\partial\left(w_{0}+\alpha w_{1}\right)}{\partial r}\right)+\frac{\partial}{\partial r} \frac{1-\alpha \vartheta}{1+\lambda 1}\left(\frac{\partial\left(w_{0}+\alpha w_{1}\right)}{\partial r}\right)-\left(M_{\sigma}^{2}+\frac{1-\alpha \vartheta}{D_{a}}\right)\left(w_{0}+\alpha w_{1}\right)+G r \vartheta+G c \varphi-\left(M_{\sigma}^{2}+\frac{1-\alpha \vartheta}{D_{a}}\right)$

We Obtain

i. $\quad$ Zero order system $\left(\alpha^{0}\right)$

$\frac{\partial p_{0}}{\partial z}=\frac{1}{r} \frac{1}{1+\lambda 1} \frac{\partial w_{0}}{\partial r}+\frac{1}{1+\lambda 1} \frac{\partial^{2} w_{0}}{\partial^{2} r}+G r \vartheta+G c \varphi-\left(M_{\sigma}^{2}+\frac{1}{D_{a}}\right) w_{0}-\left(M_{\sigma}^{2}+\frac{1}{D_{a}}\right)$,

ii. $\quad$ First order system $(\alpha)$

$\frac{\partial p_{1}}{\partial z}=-\frac{1}{r} \frac{\vartheta}{1+\lambda 1} \frac{\partial w_{0}}{\partial r}+\frac{1}{r} \frac{1}{1+\lambda 1} \frac{\partial w_{1}}{\partial r}-\frac{\vartheta}{1+\lambda 1} \frac{\partial^{2} w_{0}}{\partial^{2} r}+\frac{1}{1+\lambda 1} \frac{\partial^{2} w_{1}}{\partial^{2} r}-\left(M_{\sigma}^{2}+\frac{1}{D_{a}}\right) w_{1}+\frac{\vartheta}{D_{a}}\left(w_{0}+1\right)$,

Finally, the perturbation solutions up for $w$ are given by:

$w=w_{0}+\alpha w_{1}$,

The corresponding stream functions $u=-\frac{1}{r} \frac{\partial \psi}{\partial z}$ and $w=\frac{1}{r} \frac{\partial \psi}{\partial r}$ is $\quad \psi=\int r w d r$

The pressure rise $\Delta p$ and the friction force (at the wall) on the inner and outer tubes are $F^{(o)}$ and $F^{(i)}$, respectively, in a tube of length $L$, in their non-dimensional forms, are given by:

$\Delta p=\int_{0}^{1}\left(\frac{d p}{d z}\right) d z$

$F^{\mathrm{o}}=\int_{0}^{1} r_{2}^{2}\left(-\frac{d p}{d z}\right) d z$

$F^{\mathrm{i}}=\int_{0}^{1} r_{1}^{2}\left(-\frac{d p}{d z}\right) d z$

Substituting from equations (47\&48) in equations (55) - (57) with $r_{1}=\varepsilon, r_{2}=1+\emptyset \cdot \sin (2 \pi z)$, and then evaluating the integrations by using the language of series for several values of the parameters included, using the MATHEMATICA program, and the obtained results are discussed in the next section.

\section{Results and Discussion}

In this section, the numerical and computational results are discussed for the problem of an incompressible nonNewtonian Jeffrey fluid in a tube with heat and mass transfer through the graphical illustrations. Figure (2) shows that effects of the parameters $\epsilon$ and $R n$ on the temperature distribution function $\vartheta$ is direct, means $\vartheta$ increases with the increasing of any one of these parameters. Figure (3) shows that effects of the parameters $R e$ and $\varnothing$ on the temperature, increases with the increasing of any one of these parameters. Figure (4) It appears that the effects of $S c$ and $\epsilon$ parameters are on The distribution function of the concentration is reversed when $\mathrm{r}<1.17622$, that is $\varphi$ decreases with the increasing of $S c$ while increasing $\epsilon$ and direct when $r>1.17622$. Also $\varphi<0$ when $r<1.17622$, and $\varphi>0$ when $r>1.17622$. Figure (5). The change in concentration decreases by increasing $s r$ while increasing by $\emptyset$, when $r<1.17622$ and the direction changes when it is greater than the value. Figure (6) Shows the effects of parameters $G r$ and $M$ on the velocity distribution function w vs. $r$.It found that w increases with increase $G r$ at $r>$ 0.18 while decreases with increase $M$, and decreasing with the increase of $G r$, and $\mathrm{w}<0$ at $\mathrm{r}<0.18$ while increases with increase of $M$. Figure (7) we see that $\mathrm{w}$ is decreasing with the increase of $M$, when $\mathrm{r}<0.18$ then the $D a$ decreases, and $\mathrm{w}$ is decreases with increase of $M$ when $\mathrm{r}>0.18$ the $D a$ is also in conflict with the $M$. Figure (8) w increases with increase of $S r$ and $S c$ when $\mathrm{r}<0.18$, and w decreases with $S r$ and $S c$ increase when $\mathrm{r}>0.18$. Figure (9) we see that $\mathrm{w}$ is decreasing with the increase of $\lambda 1$,when $\mathrm{r}<0.18$ then the $\epsilon$ decreases, and $\mathrm{w}$ is decreases with increase of $\lambda 1$ when $r>0.18$ the $\epsilon$ is also in conflict with the $\lambda 1$. Figure (10) shows the effects of parameters $\lambda 1$ and $G c$ on $d p / d z$ vs. z. It was found that $d p / d z$ increases with increasing each $\lambda 1$ and Gc. Figure (11) we see that $d p / d z$ decreases with an increase of q2 and $\epsilon$, while increases with increasing of $M$. Figure (12) decreases $d p / d z$ with increase for each $D a$ and Gr. Figure (13) increases $d p / d z$ with increase for each $\phi$ and $\epsilon$. Figure (14) illustrates the effects of the parameters $\epsilon$ and $G c$ on the pressure rise $\Delta p$ versus $G r$ respectively, shows that the 
variation of $\Delta p$ vs. $G r$, it is found that $\Delta p$ decreases with the increasing for each $\epsilon$ and Gc. Figure (15) we see that $\Delta p$ vs. $\phi$. It is found $\Delta p$ increases with the increasing of $D a$ while decreases with an increase of $G r$. Figure (16) shows that the variation of $F^{(i)}$ vs. $G r$. It is found that $F^{(i)}$ decreases with the increasing for $\epsilon$. Figure (17) shows that the variation of $F^{(i)}$ vs. $\phi$. It is found $F^{(i)}$ decreases with the increasing Da while increases with an increase of $G r$, and it changes its direction when it is $\phi<0.07$. Figure (18) shows that the variation of $F^{(0)}$ vs. Gr. It is found that $F^{(0)}$ decreases with the increasing for $\epsilon$. Figure (19) shows that the variation of $F^{(0)}$ vs. $\phi$. It is found $F^{(0)}$ decreases with the increasing Da while increase with an increasing of $G r$, and it changes its direction when it is $\phi<0.07$. Figure (20) we observe the increase in $\phi$ and the number of valves increases gradually. Figure (21) the bracelets grow when the $D a$ increases. Figure (22) the number of bracelets is lower M when the value is reduced. Figure (23) By increasing the value of $\epsilon$, the wheels are increasing.

\section{Concluding Remarks}

We discuss the Influence of varying temperature and concentration on (MHD) peristaltic transport for Jeffrey fluid with variable viscosity through porous channel. We found the velocity and temperature are analytical. We used different values to find the results of pertinent parameters, namely for the velocity and temperature. The key point is listed below:

i. We show that by increasing $R n, R e$ and $\epsilon$ the temperature $\vartheta$ increasing and the temperature $\vartheta$ decreases with the increasing $\varnothing$.

ii. We show that by increasing $S r$ and $S c$ the concentration $\varphi$ increasing when $r>1.3$ and the concentration $\varphi$ decreases with the increasing $S r$ and $S c$ when $r<1.3$.

iii. The velocity profiles were increased by the increasing $S r, S c, \sigma$ and $M$. when $r<0.25$, and the velocity decreased when $r>0.25$.

iv. The velocity profiles were decreased by the increasing $G r, \epsilon, \lambda 1$ and $D a$ when $r<0.25$, and the velocity increased when $r>0.25$.

v. $\quad \frac{d p}{d z}$ increased by the increasing parameters $G r, \epsilon, \phi, \lambda 1$ and Gc.

vi. $\quad \frac{d p}{d z}$ decreased by the increasing parameters $q 2$ and $D a$.

vii. $\quad \Delta p$ Increased by the increasing $\lambda 1$ and decreased by the increasing $G r$ and $\Delta p$ increased by the increasing Gr and $D a$, when $\phi<0.37$, and decreased when $\phi>0.37$.

viii. $\quad F^{i}$ decreased by the increasing $\lambda 1$ and increased by the increasing $G r$ and $F^{i}$ decreased by the increasing Gr and $D a$, when $\phi<0.37$, and increased when $\phi>0.37$.

ix. $\quad F^{o}$ decreased by the increasing $\lambda 1$ and increased by the increasing $G r$ and $F^{o}$ decreased by the increasing $G r$ and $D a$, when $\phi<0.37$, and increased when $\phi>0.37$. 


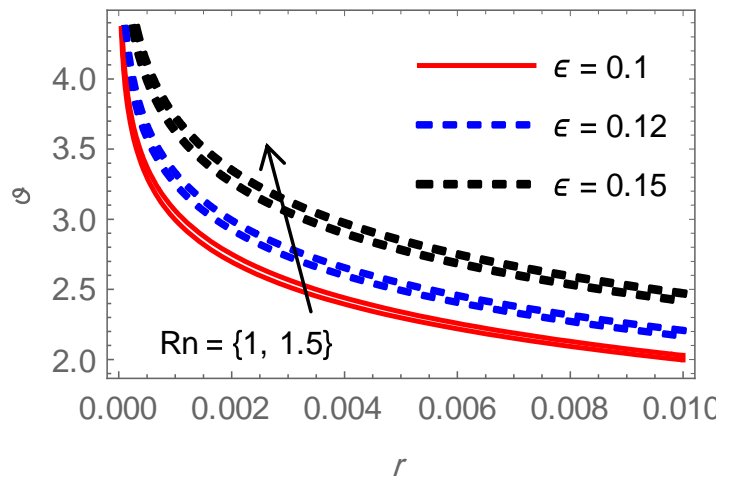

Fig. (2): The variation of temperature $\vartheta$ vs. $r$ at Fig. (3): The variation of temperature $\vartheta$ vs. $r$ at $\Omega=1, \operatorname{Re}=0.9, \operatorname{Pr}=1, \emptyset=0.3, z=0.1$.

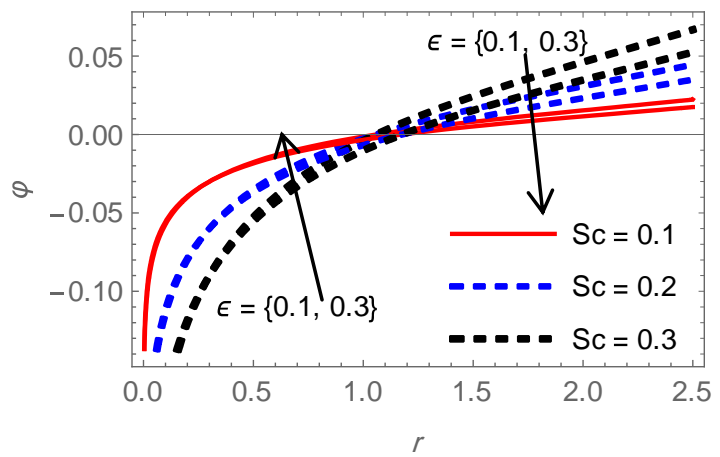

Fig. (4): The variation of concentration $\varphi$ vs. $r$ at $\Omega=1, \operatorname{Re}=3, \operatorname{Pr}=2, \operatorname{Rn}=0.5, z=0.1, \varnothing=0.3, \mathrm{Sr}=$ 0.3 .

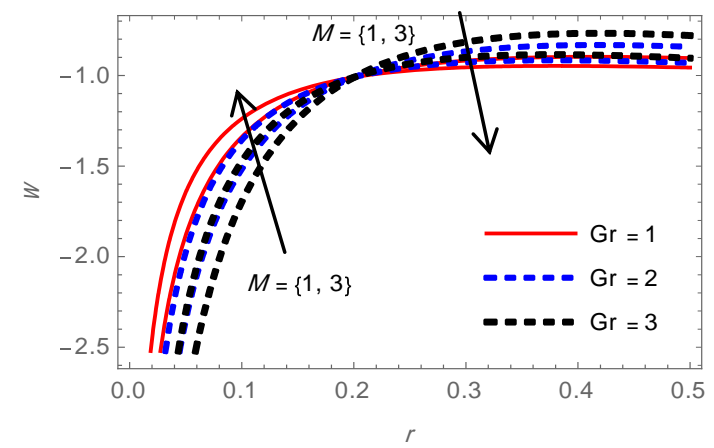

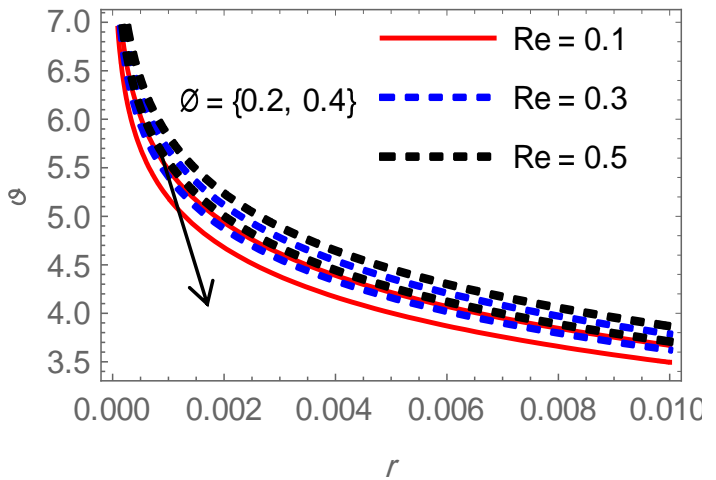
$\Omega=1, \operatorname{Pr}=1, \epsilon=0.3, R n=2, z=0.1$.

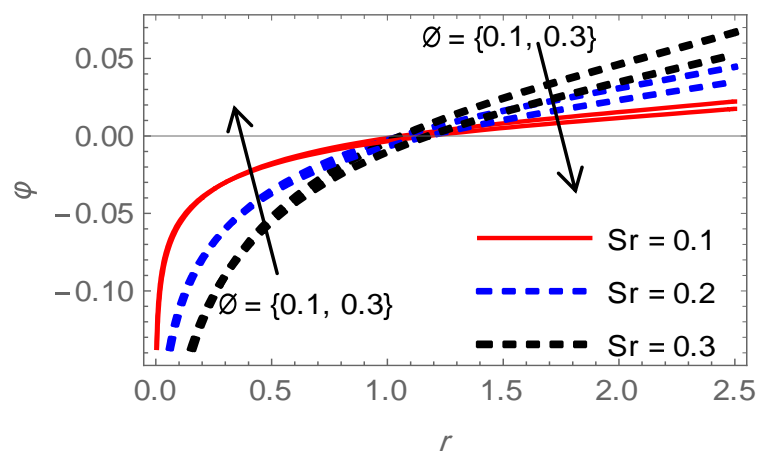

Fig. (5): The variation of concentration $\varphi$ vs. $r$ $\Omega=1, \operatorname{Re}=3, \operatorname{Pr}=2, \operatorname{Rn}=0.5, z=0.1, \mathrm{Sc}=$ $0.3, \epsilon=0.3$.

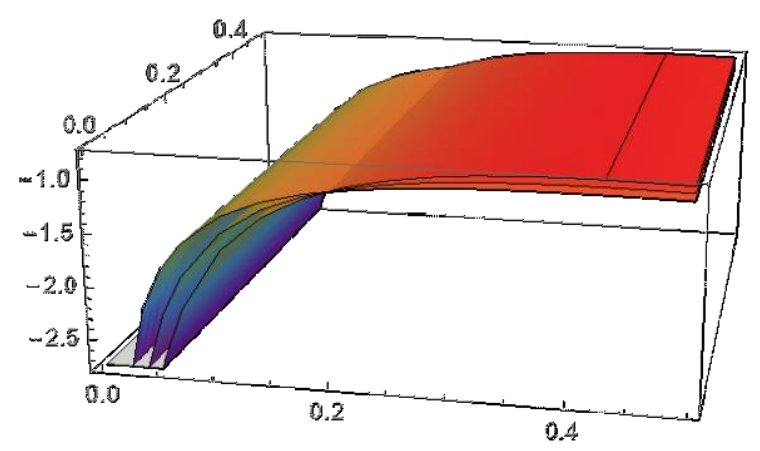

Fig. (6):Velocity distribution $w$ at $\Omega=0.9, \phi=0.3, \sigma=\frac{\pi}{4}, z=0.01, \epsilon=0.2, \mathrm{Da}=0.9, \lambda 1=0.1, \operatorname{Re}=1, \mathrm{Rn}=$ $2, \operatorname{Pr}=2, \mathrm{Gc}=1, \mathrm{q} 2=0.5, \mathrm{Sr}=0.1, \mathrm{Sc}=0.5, \epsilon=0.2$.
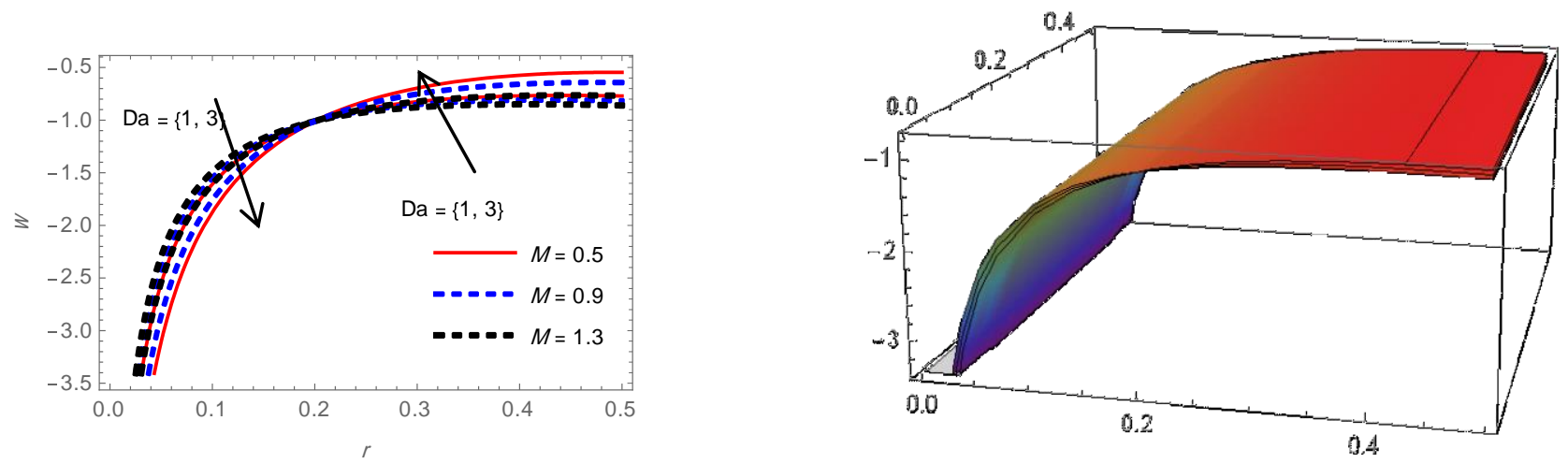

Fig. (7):Velocity distribution $\quad w \quad$ at $\quad \Omega=0.9, \quad \phi=0.3, \sigma=\frac{\pi}{4}, z=0.01, \epsilon=0.2, \mathrm{Gr}=2, \quad \lambda 1=0.1, \operatorname{Re}=1, \mathrm{Rn}=$ 
$2, \operatorname{Pr}=2, \mathrm{Gc}=1, \mathrm{q} 2=0.5, \mathrm{Sr}=0.1, \mathrm{Sc}=0.5, \epsilon=0.2$.
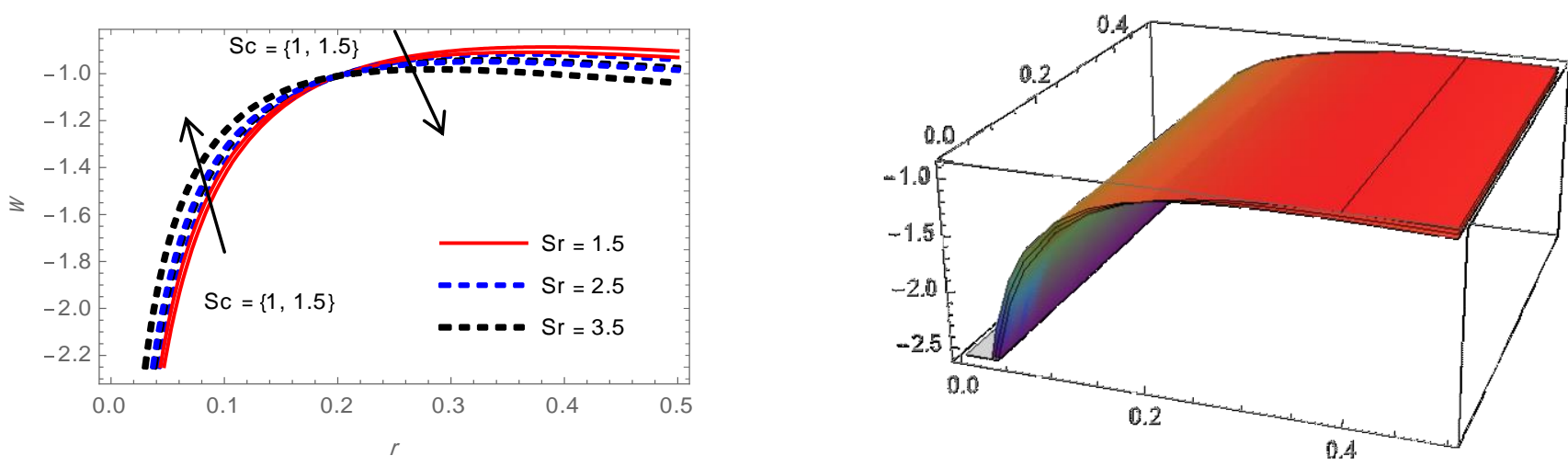

Fig. (8): Velocity distribution $w$ at $\Omega=0.9, \phi=0.3, \sigma=\frac{\pi}{4}, z=0.01, \epsilon=0.2, \mathrm{Da}=0.9, \lambda 1=0.1, \operatorname{Re}=1, \mathrm{Rn}=$ $2, \operatorname{Pr}=2, G r=2, \mathrm{Gc}=1, M=1.1, \mathrm{q} 2=0.5, \epsilon=0.2$.
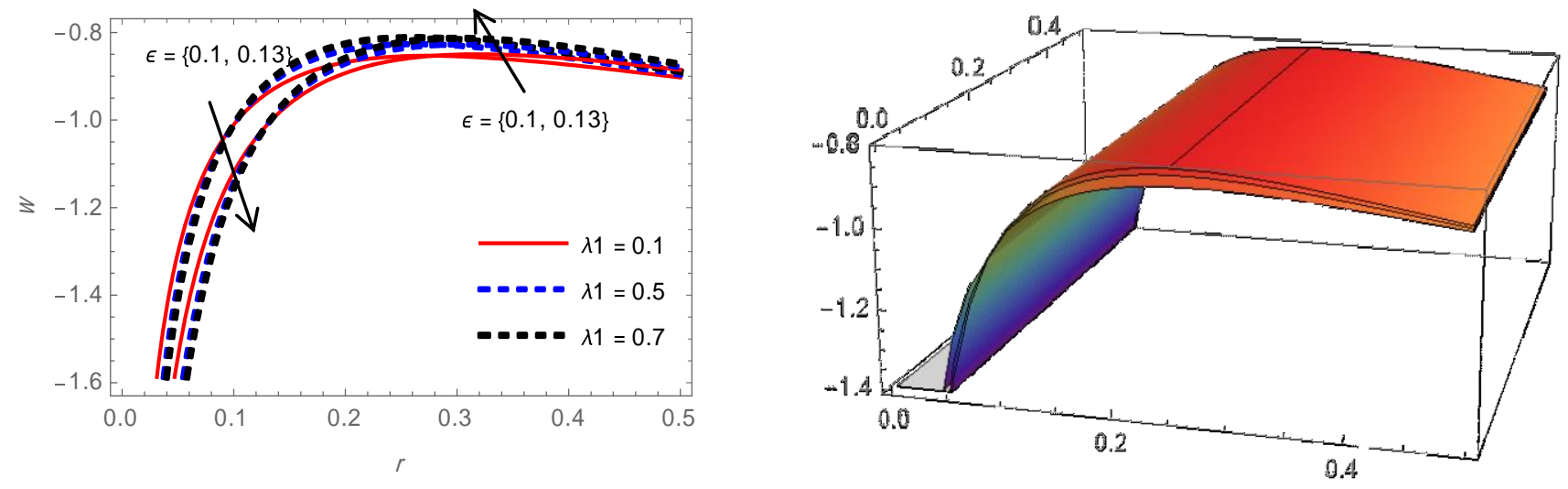

Fig. (9): Velocity distribution $w$ at $\Omega=0.9, \phi=0.3, \sigma=\frac{\pi}{4}, z=0.01, S c=0.5, \mathrm{Da}=0.9, \mathrm{Sr}=0.1, \mathrm{Re}=1, \mathrm{Rn}=$ $2, \operatorname{Pr}=2, G r=2, \mathrm{Gc}=1, M=1.1, \mathrm{q} 2=0.5$.
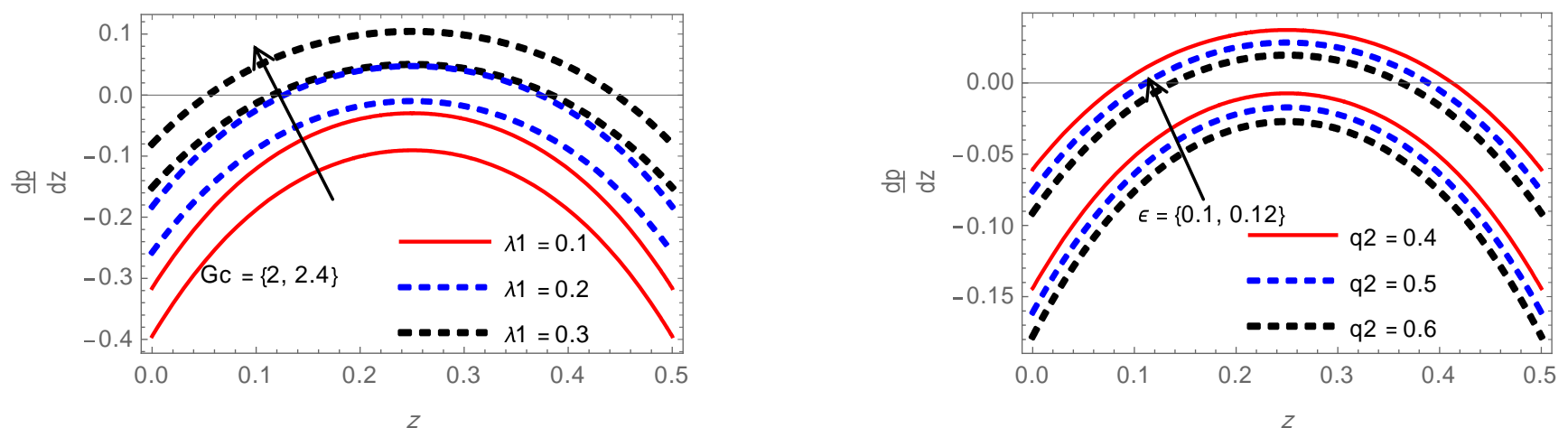

Fig. (10): The variation of $\frac{d p}{d z}$ vs. $\mathrm{z}$ at $\Omega=0.9, \operatorname{Re}=1$, Fig. (11): The variation of $\frac{d p}{d z} \mathrm{vs.} \mathrm{z}$ at $\Omega=0.9, \operatorname{Re}=$ $\operatorname{Pr}=2, \mathrm{Da}=0.9, \sigma=\frac{\pi}{4}, z=0.01, \mathrm{Rn}=2, G r=2, \epsilon=\quad 1, \operatorname{Pr}=2, \mathrm{Da}=0.9, \sigma=\frac{\pi}{4}, z=0.01, \mathrm{Rn}=2, M=$ 0.2 ,

$1.1, \mathrm{Gr}=2 . \mathrm{Sc}=0.5, \mathrm{Sr}=0.1$. 


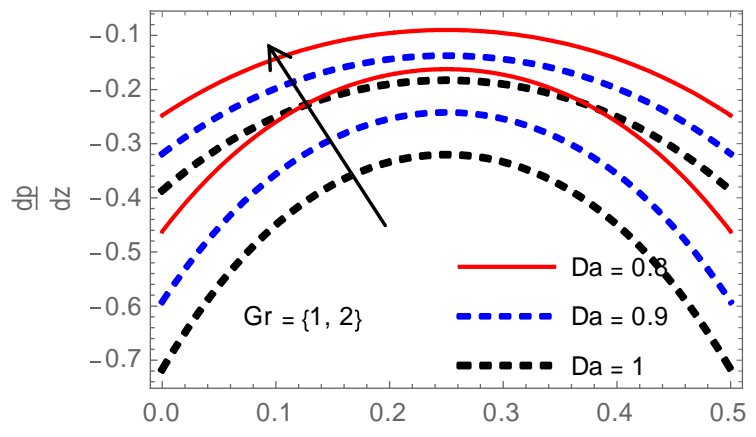

Fig. (12): The variation of $\frac{d p}{d z}$ vs. z at $\Omega=0.9, R e=1$, $\operatorname{Pr}=2, \sigma=\frac{\pi}{4}, \mathrm{Rn}=2, G c=1, \lambda 1=0.1, \epsilon=0.2, \mathrm{Sc}=$ $0.5, \mathrm{Sr}=0.1$.

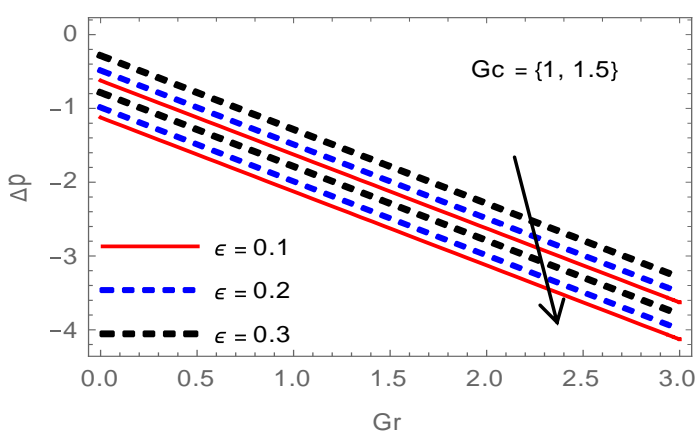

Fig. (14): The variation of $\Delta p \mathrm{vs} G r, \Omega=0.9, \mathrm{Rn}=$ $2, \operatorname{Pr}=2, \quad \mathrm{Sc}=0.5, \mathrm{Sr}=0.1, \mathrm{Da}=0.9, M=1.1, \sigma=$ $\frac{\pi}{4}, z=0.01$.

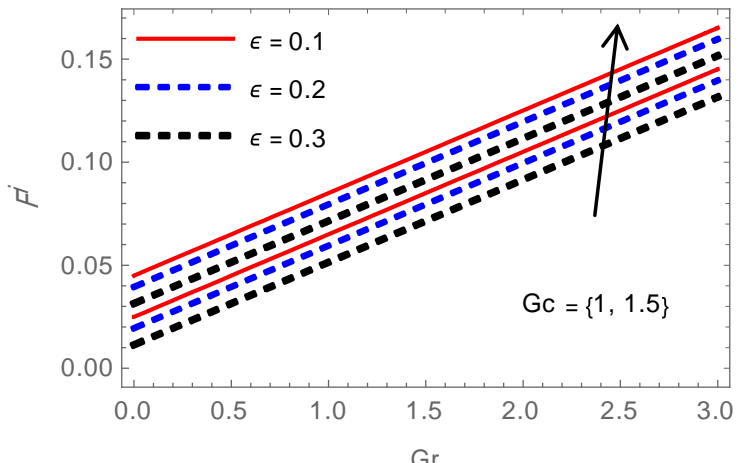

Fig. (16): The variation of $F^{i}$ vs. $G r, \Omega=0.9, \mathrm{Rn}=2, P r=$ $2, \mathrm{Sc}=0.5, \mathrm{Sr}=0.1, \mathrm{Da}=0.9, M=1.1, \sigma=\frac{\pi}{4}, z=0.01$.

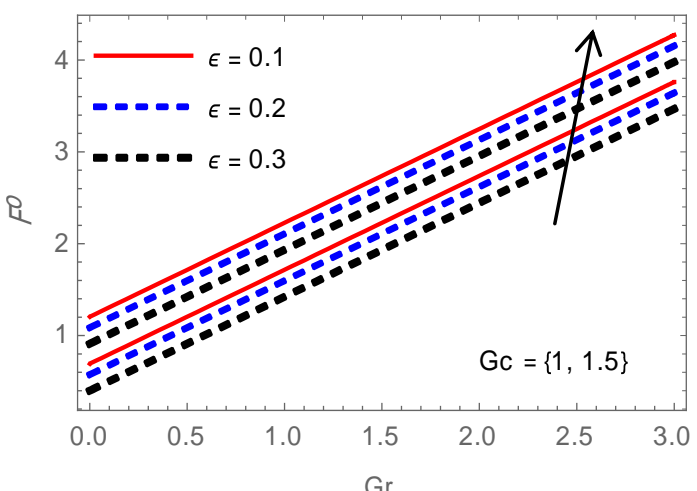

Fig. (18): The variation of $F^{0}$ vs. $G r, \Omega=0.9, \mathrm{Rn}=$ $2, \operatorname{Pr}=2, \mathrm{Sc}=0.5, \mathrm{Sr}=0.1, \mathrm{Da}=0.9, M=1.1, \sigma=$ $\frac{\pi}{4}, z=0.01$.

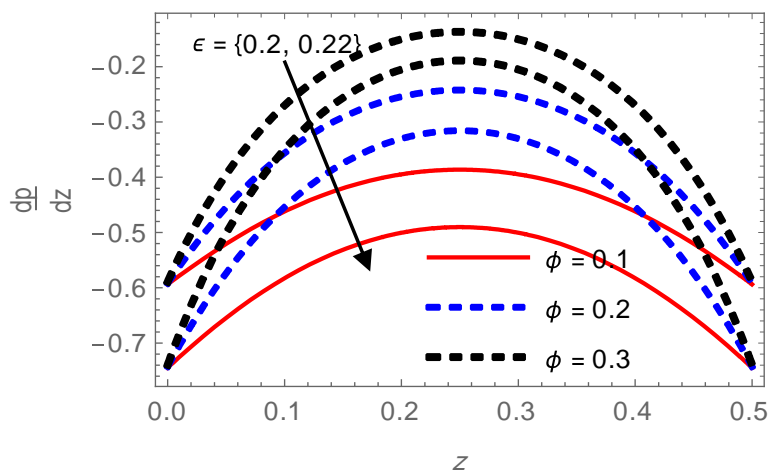

Fig. (13): The variation of $\frac{d p}{d z}$ vs. $\mathrm{z}$ at $\Omega=0.9, \operatorname{Re}=1, \operatorname{Pr}=$ 2, $\mathrm{Da}=0.9, \sigma=\frac{\pi}{4}, \mathrm{Rn}=2, G c=1, \lambda 1=0.1, \mathrm{Sc}=0.5, \mathrm{Sr}=$ 0.1 .

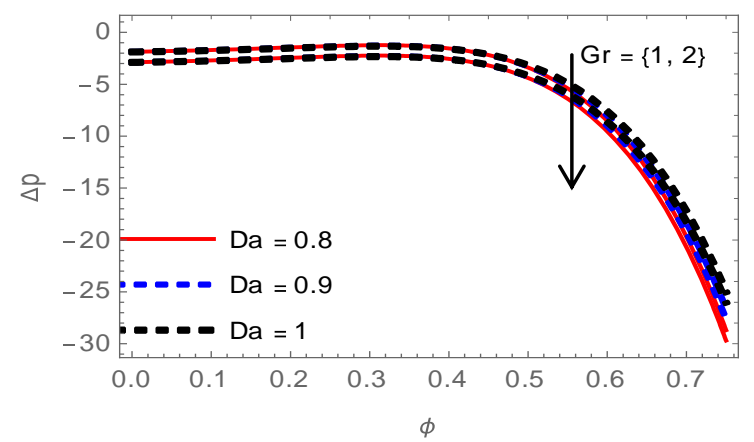

Fig. (15): The variation of $\Delta p \mathrm{vs} \phi, \Omega=0.9, \mathrm{Rn}=$ $2, \operatorname{Pr}=2, \mathrm{Sc}=0.5, \mathrm{Sr}=0.1, M=1.1, \mathrm{Gc}=1, \epsilon=$ $0.2, \sigma=\frac{\pi}{4}, z=0.01$.

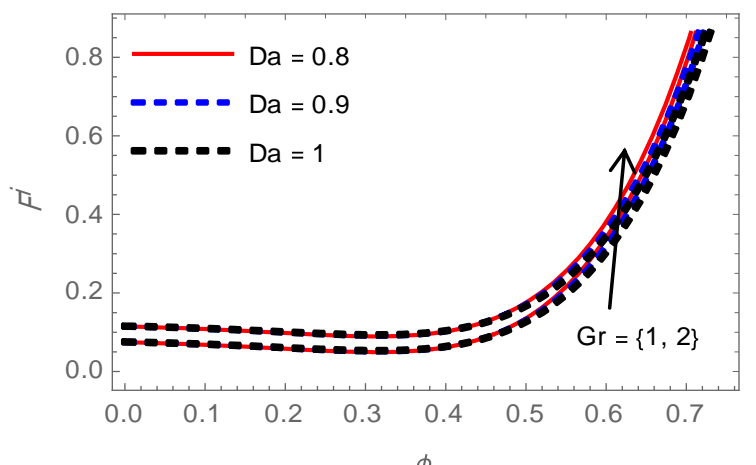

Fig. (17): The variation of $F^{i}$ vs. $\phi, \Omega=0.9, \mathrm{Rn}=2, \operatorname{Pr}=2$, $\mathrm{Sc}=0.5, \mathrm{Sr}=0.1, M=1.1, \mathrm{Gc}=1, \epsilon=0.2, \sigma=\frac{\pi}{4}, z=0.01$.

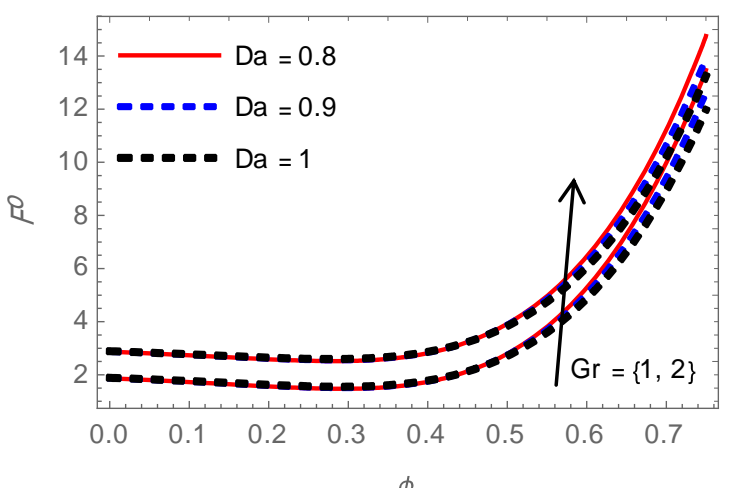


Fig. (19): The variation of $F^{0}$ vs. $\phi, \Omega=0.9, \mathrm{Rn}=$

$2, \operatorname{Pr}=2, \mathrm{Sc}=0.5, \mathrm{Sr}=0.1, \mathrm{Da}=0.9, M=1.1, \sigma=\frac{\pi}{4}, z=$
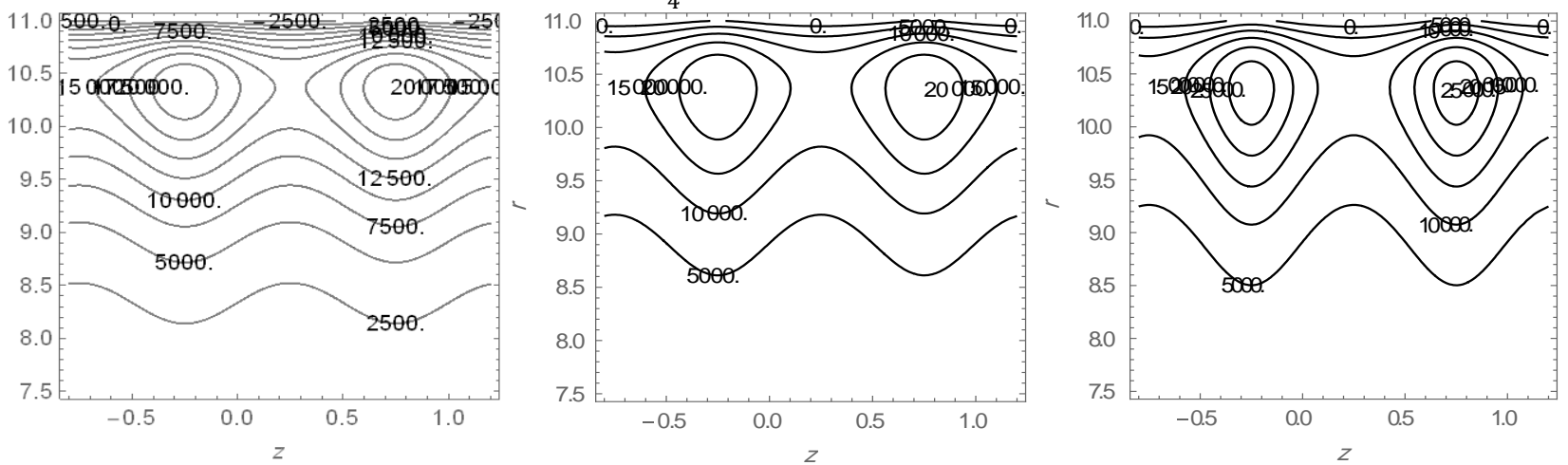

Fig. (20) Streamlines for $\phi$ when $\Omega=0.9, \epsilon=0.2, \lambda 1=0.1, \operatorname{Re}=1$, Rn $=2$, q $2=0.5$, Sc $=0.5$, S1 $=0.1$, Gc $=2, M=$ $1.1, \mathrm{Da}=0.9, \sigma=\frac{\pi}{4}, i=$.
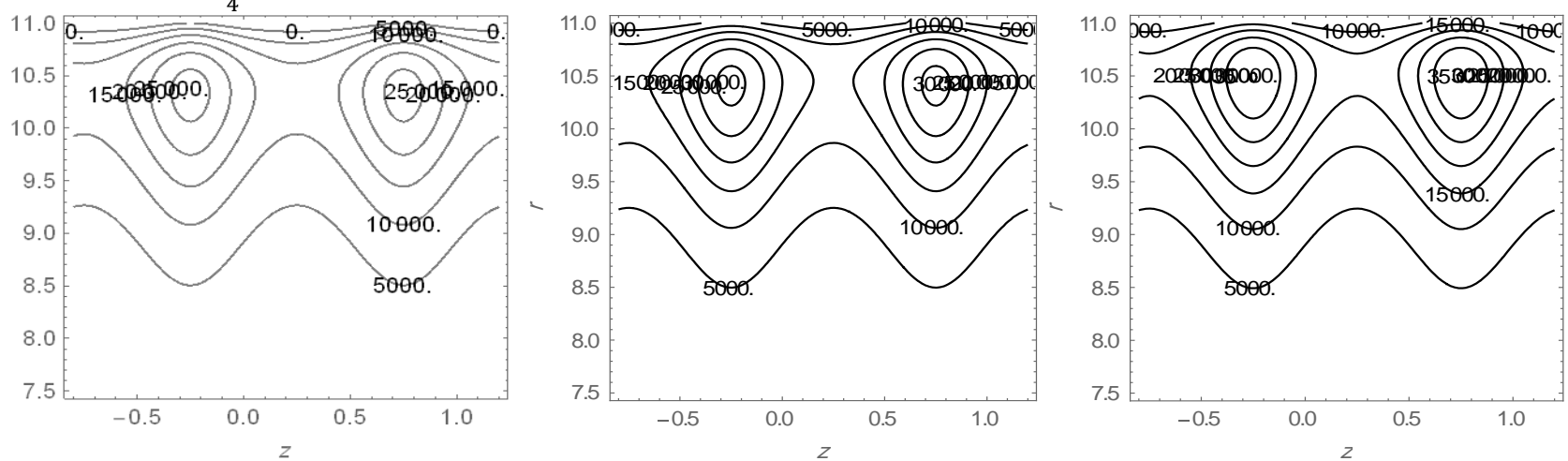

Fig. (21) Streamlines for $D a$ when $\Omega=0.9, \epsilon=0.2, \phi=0.2, \lambda 1=0.1, R e=1$, Rn $=2$, q $2=0.5, \mathrm{Sc}=0.5, \mathrm{~S} 1=$ $0.1, \mathrm{Gc}=2, M=1.1, \mathrm{Da}=0.9, \sigma=\frac{\pi}{4}$.
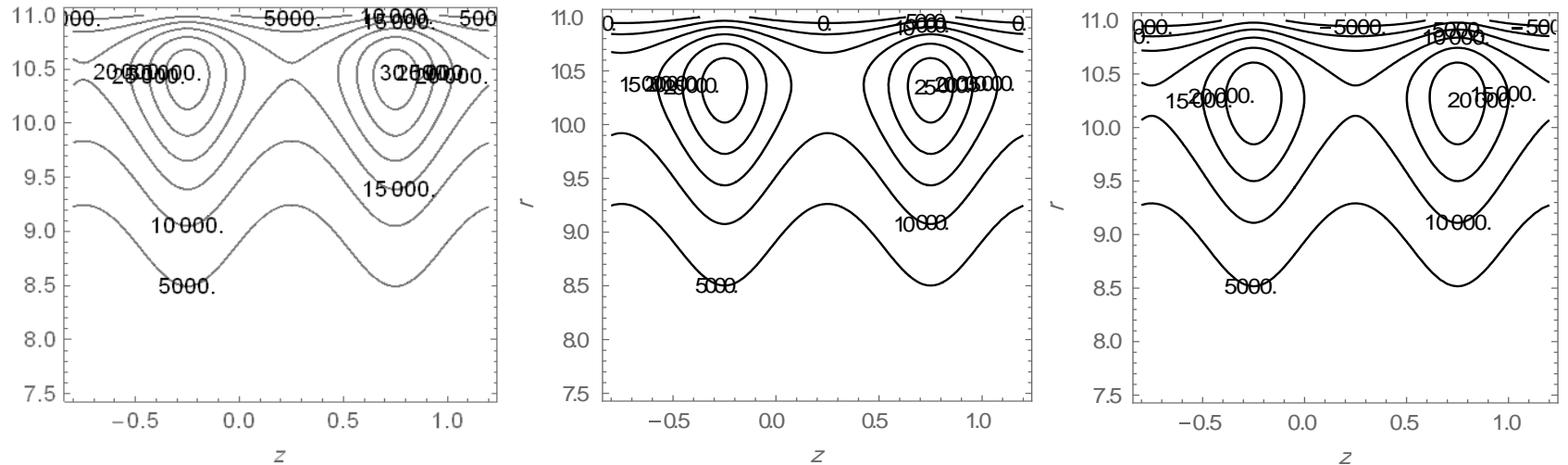

Fig. (22) Streamlines for $M$ when $\Omega=0.9, G r=1, \phi=0.2, \lambda 1=0.1, \operatorname{Re}=1$, Rn $=2, \mathrm{q} 2=0.5$, $\mathrm{Sc}=0.5, \mathrm{~S} 1=0.1, \mathrm{Gc}=2, M=$ $1.1, \mathrm{Da}=0.9, \sigma=\frac{\pi}{4}$.
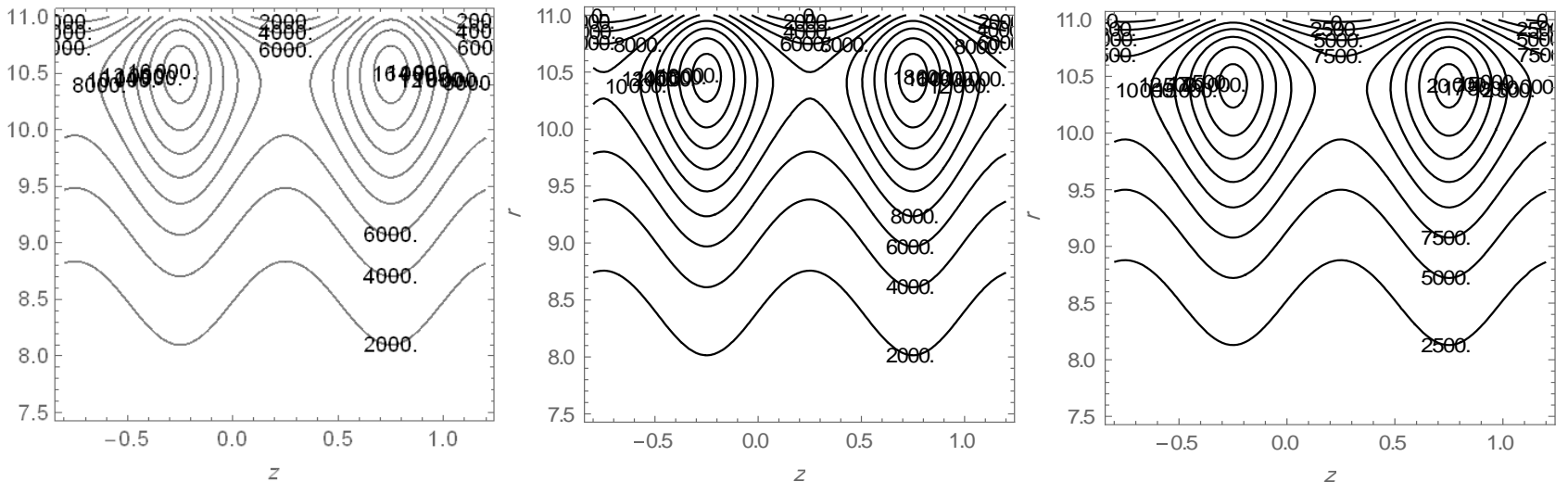

Fig. (23) Streamlines for $\epsilon$ when $\Omega=0.9, G r=1, \phi=0.2, \lambda 1=0.1, R e=1$, Rn $=2$, q $2=0.5$, Sc $=0.5$, S1 $=$ $0.1, \mathrm{Gc}=2, M=1.1, \mathrm{Da}=0.9, \sigma=\frac{\pi}{4}$. 


\section{Reference}

[1] D. G. S. Al-khafajy, "Influence of MHD and Wall Properties on the Peristaltic Transport of a Williamson Fluid with Variable Viscosity Through Porous Medium," Iraqi J. Sci., 58( 2),( 2017), 1076-1089.

[2] M.Hameed and S.Nadeem, "Unsteady MHD flow of a non-Newtonian fluid on a porous plate," Journal of Mathematical Analysis and Applications, vol. 325, no. 1, (2007), pp. 724-733.

[3] J. D. Kevorkian, Jirair, Cole, Perturbation Methods in Applied Mathematics. Springer-Verlag New York, (1981).

[4] A. A. Khan, R. Ellahi, and K. Vafai, "Peristaltic Transport of a Jeffrey Fluid with Variable Viscosity through a Porous Medium in an Asymmetric Channel," vol. (2012).

[5] W. S. Khudair and D. G. S. Al-khafajy, Influence of heat transfer on Magneto hydrodynamics oscillatory flow for Williamson fluid through a porous medium, Iraqi J. Sci., vol. 59, no. 1B, (2018), pp. 389-397.

[6] M. Y.Malik, A. Hussain, and S. Nadeem, "Flow of a Jeffrey-six constant fluid between coaxial cylinders with heat transfer," Communications in Theoretical Physics, vol. 56, (2011), pp. 345-351.

[7] S. Nadeem, N. S. Akbar, and M. Y. Malik, "Numerical solutions of peristaltic flow of a newtonian fluid under the effects ofmagnetic field and heat transfer in aporous concentric tubes," Zeitschrift fur Naturforschung A, vol. 65, no. 5, (2010), pp. 369-380.

[8] S.Nadeem and M. Awais, "Thin film flow of an unsteady shrinking sheet through porous medium with variable viscosity," Phys. Lett. A,vol. 372, (2008), pp.4965-4972.

[9] S.Srinivas and R. Gayathri, "Peristaltic transport of a Newtonian fluid in a vertical asymmetric channel with heat transfer and porous medium," AppliedMathematics and Computation, vol. 215, no. 1, (2009), pp. 185-196.

[10] S.Srinivas and M. Kothandapani, "The influence of heat and mass transfer on MHD peristaltic flow through a porous space with compliant walls," Applied Mathematics and Computation, vol. 213, no. 1(2009), pp. 197-208,.

[11] W. Tan and T. Masuoka, "Stokes' first problem for a second grade fluid in a porous half-space with heated boundary," International Journal of Non-Linear Mechanics, vol. 40, no. 4, (2005), pp. 515-522.

[12] M.Vidhya , Niranjana N., Govindarajan A; Free Convective and Oscillatory Flow of a Dusty Fluid Through a Porous Medium, International Journal of Pure and Applied Mathematics, 114(3),(2017), pp. 445-456. 